\title{
ESTRATIGRAFIA RASA DA ILHA COMPRIDA (SP): UM EXEMPLO DE APLICAÇÃO DO GPR
}

\author{
Otávio C. B. Gandolfo ${ }^{1}$, Luiz A. P. Souza ${ }^{2}$, Moysés G. Tessler ${ }^{3}$ \& Marcelo \\ Rodrigues $^{3}$
}

Received May 24, 2001 / Accepted May 30, 2003

\begin{abstract}
A estratigrafia rasa da Ilha Comprida, localizada no litoral sul do Estado de São Paulo, foi analisada em detalhe através de perfis de radar solo penetrante (GPR), executados ao longo da estrada que corta transversalmente a ilha em sua porção extremo sudeste. O GPR constitui-se num método geofísico de alta resolução, com vasta aplicação em estudos de áreas costeiras. Registros de excelente qualidade obtidos neste estudo possibilitaram a visualização de características da estratigrafia rasa da Ilha Comprida, que foram correlacionadas ao modelo evolutivo descrito para a área. A interpretação dos dados revelou a ocorrência de três unidades estratigráficas, separadas por contatos bastante conspícuos identificados nos registros. As duas unidades superiores corresponderiam a depósitos holocênicos, formados em eventos transgressivo e regressivo, sendo este último responsável pela formação de cordões arenosos claramente identificados nos registros. Estes depósitos encontram-se superpostos, em contato erosivo, a sedimentos associados a variações do nível médio do mar no Pleistoceno. Os resultados mostram uma evidente correlação entre as unidades identificadas nas seções de GPR e o modelo evolutivo sedimentar proposto por Suguio \& Martin (In Rabassa, J. (Ed.) Quarternary of S. America and Antartica Península, 1978), baseado nas variações do nível médio do mar no Quaternário, para a região sul da Ilha Comprida.
\end{abstract}

Palavras-chave: GPR; Radar solo penetrante; Estratigrafia rasa; Quaternário; Ilha Comprida.

SHALLOW STRATIGRAPHY OF ILHA COMPRIDA -SP BY GROUND PENETRATING RADAR SURVEY - The shallow stratigraphy of Ilha Comprida-SP was analised based on ground penetrating radar (GPR) profiles, that allowed to identify some important geological characteristics of southern São Paulo State coast. GPR is a very high resolution geophysical method applied in shallow investigations providing excellent results in several geologic environments. The interpretation of the data revealed the occurrence of three main stratigraphic units separated by strong electromagnetic reflectors. The two superior units can be correlated to the holocenic deposits, formed in transgressive and regressive events. The deeper unit is related to the sandy ridges formation, clearly identified in the registers. These deposits overlap, in erosive contact, the sediments associated with sea level changes in the Pleistocene. These results show an evident correlation between the stratigraphic units identified in the GPR sections and the sedimentary evolutive model, proposed by Suguio \& Martin (In Rabassa, J. (Ed.) Quaternary of S. America and Antartica Peninsula, 1978), based in the sea level changes during the Quaternary, for the southern region of the Ilha Comprida.

Key words: GPR; Shallow stratigraphy; Shallow geophysics; Quaternary; Ilha Comprida.

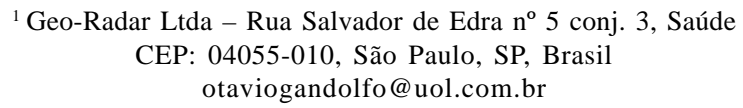

${ }^{2}$ Instituto de Pesquisas Tecnológicas do Estado de São Paulo - IPT

Av. Prof. Almeida Prado 532, Cidade Universitária, CEP: 05508-901, São Paulo, SP, Brasil laps@ipt.br

\footnotetext{
${ }^{3}$ Instituto Oceanográfico da Universidade de São Paulo -IO-USP

Praça do Oceanográfico, 191 - Butantã, CEP: 05508-900, São Paulo, SP, Brasil mgtessle@usp.br mrrodrig@usp.br
} 


\section{INTRODUÇÃO}

O Radar Penetrante no Solo (GPR) é o método geofísico mais indicado para investigações rasas, apresentando excelentes resultados em determinados tipos de ambientes geológicos. As ondas eletromagnéticas empregadas possibilitam a visualização detalhada das camadas próximas à superfície diferenciando-o dos demais métodos geofísicos. Outra característica que destaca este método geofísico, frente a outras formas de investigação rasa, é a enorme versatilidade operacional (equipamento leve e portátil) que permite a aquisição de grande quantidade de dados num curto intervalo de tempo, viabilizando desta forma uma amostragem espacial bastante detalhada. Fisher et al., 1992, destaca também como importante propriedade deste método geofísico de investigação rasa, as similaridades existentes com a sísmica de reflexão o que permite que rotinas do processamento de dados sísmicos possam ser utilizadas, com adaptações relativamente simples, no processamento de dados GPR.

Exemplos de mapeamento estratigráfico através da utilização do GPR em vários tipos de ambientes geológicos são encontrados em Beres \& Haeni (1991). Particularmente, excelentes resultados podem ser obtidos em levantamentos sobre depósitos arenosos, conseqüência da baixa atenuação sofrida pelo sinal eletromagnético neste meio (Jol \& Smith, 1991; Jol et al., 1996; Van Overmeeren, 1998; Daly et al., 2002).

O objetivo principal deste trabalho foi analisar a estratigrafia rasa da Ilha Comprida tendo como base os dados obtidos com o método geofísico de alta resolução denominado de radar solo penetrante (GPR), correlacionando os resultados desta análise com o modelo evolutivo proposto por Suguio \& Martin (1978). Secundariamente, este trabalho teve por objetivo, evidenciar a enorme potencialidade de aplicação deste método geofísico de alta resolução em estudos estratigráficos rasos e de detalhes em áreas costeiras.

\section{ASPECTOS GERAIS DA ÁREA INVESTIGADA}

A Ilha Comprida se constitui em uma feição arenosa alongada localizada no litoral sul do Estado de São Paulo, entre as latitudes $24^{\circ} 45^{\prime}$ e $25^{\circ} 05^{\prime} \mathrm{S}$ e longitudes $47^{\circ} 30^{\prime}$ e $47^{\circ} 55^{\prime} \mathrm{W}$. Possui cerca de 72 $\mathrm{km}$ de extensão e larguras variando entre 2 e $5 \mathrm{~km}$, com altitudes inferiores a 7 metros, estando separada do continente por canais lagunares pertencentes ao Sistema Cananéia - Iguape, desenvolvidos à retaguarda da Ilha Comprida (Fig.1).

A partir de levantamentos aerofotogramétricos executados por Barcelos (1975) e Suguio \& Martin (1978), foram observadas ao longo de toda a superfície da feição arenosa a presença de cordões arenosos, com até $65 \mathrm{~km}$ de extensão, predominantemente paralelos a atual linha de costa.

Os sedimentos destes cordões são constituídos principalmente por areias finas a muito finas (> 98\%), onde o quartzo se apresenta como o mineral predominante (>95\%).

Os cordões arenosos acham-se preferencialmente agrupados em duas faixas distintas do terreno sendo uma mais interna, ou correspondente a área atualmente mais próximas dos canais lagunares e outra mais externa, próxima à face oceânica. Estas faixas de cordões arenosos estão separadas por uma área de baixa altitude com cerca de 100 metros de largura, e cerca de $50 \mathrm{~km}$ de extensão, ocupada por sedimentos areno-argilosos de ambiente paludal.

De acordo com o modelo proposto por Suguio \& Martin (1978), a Ilha Comprida tem sua gênese e evolução associadas as variações relativas do nível do mar Quaternário, e aos mecanismos de dinâmica litorânea holocênica, atuantes ao longo de toda a região costeira rasa do litoral sul do Estado de São Paulo, originadas pelos sistemas de ondas incidentes (Fig. 2).

A partir de um núcleo de idade mais antiga (pleistocênica), situado a SW da Ilha, a feição arenosa evoluiu ao longo dos últimos 5.100 anos, em duas etapas diretamente vinculadas aos mecanismos de oscilação relativa do nível do mar, identificados por Suguio \& Martin (1978).

Na primeira etapa, após o máximo transgressivo holocênico (5.100 anos), a Ilha estendeu-se rumo a norte, em direção a Iguape, impulsionada pelo sentido resultante da dinâmica de correntes litorânea dos sistemas de ondas incidentes na área. Neste alongamento é formado nas áreas a NE da Ilha, o canal lagunar que separa a Ilha Comprida do 


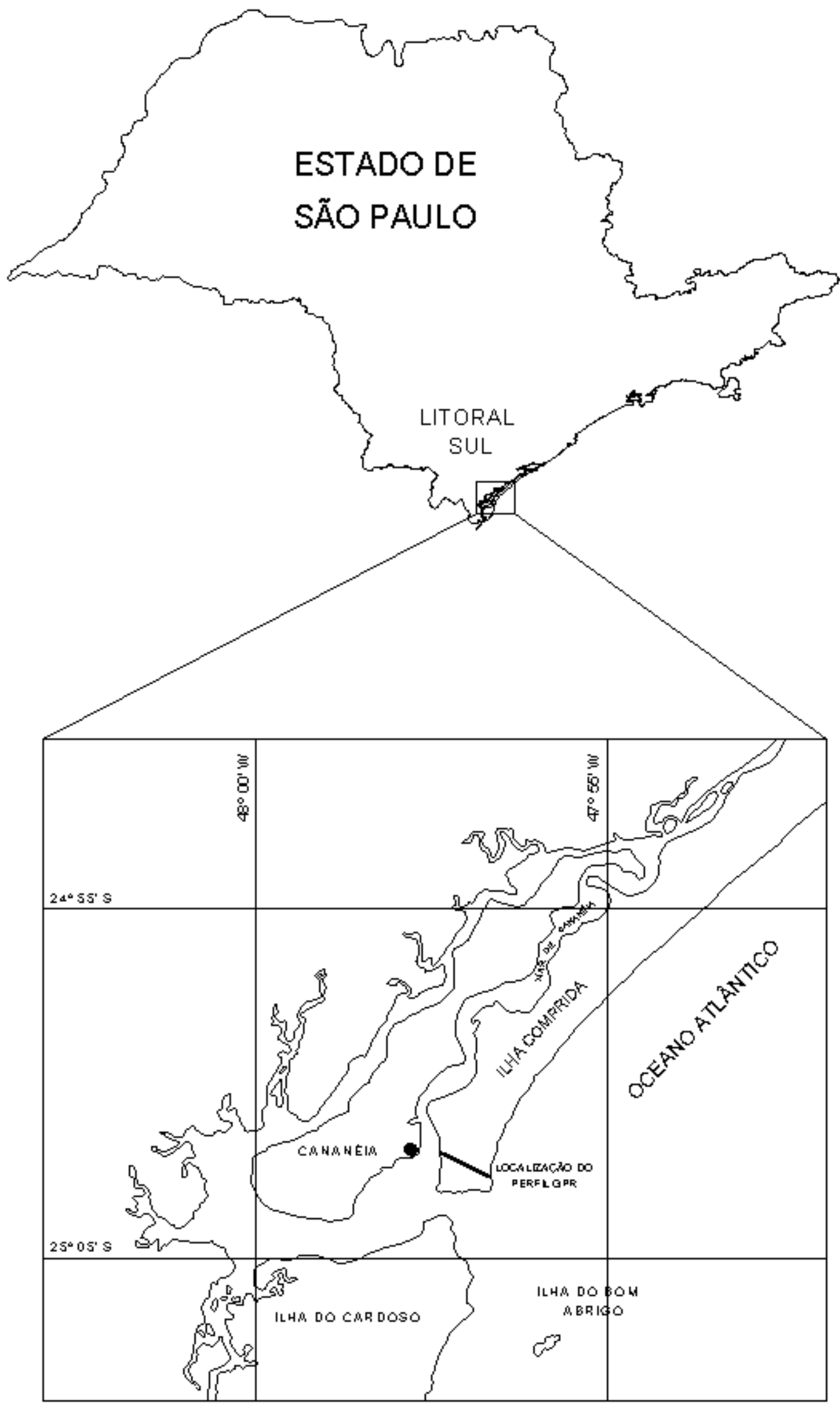

Figura 1: Mapa de localização da área estudada e dos perfis de GPR realizados.

Figure 1: Map of the studied area with the site of the GPR profiles. 


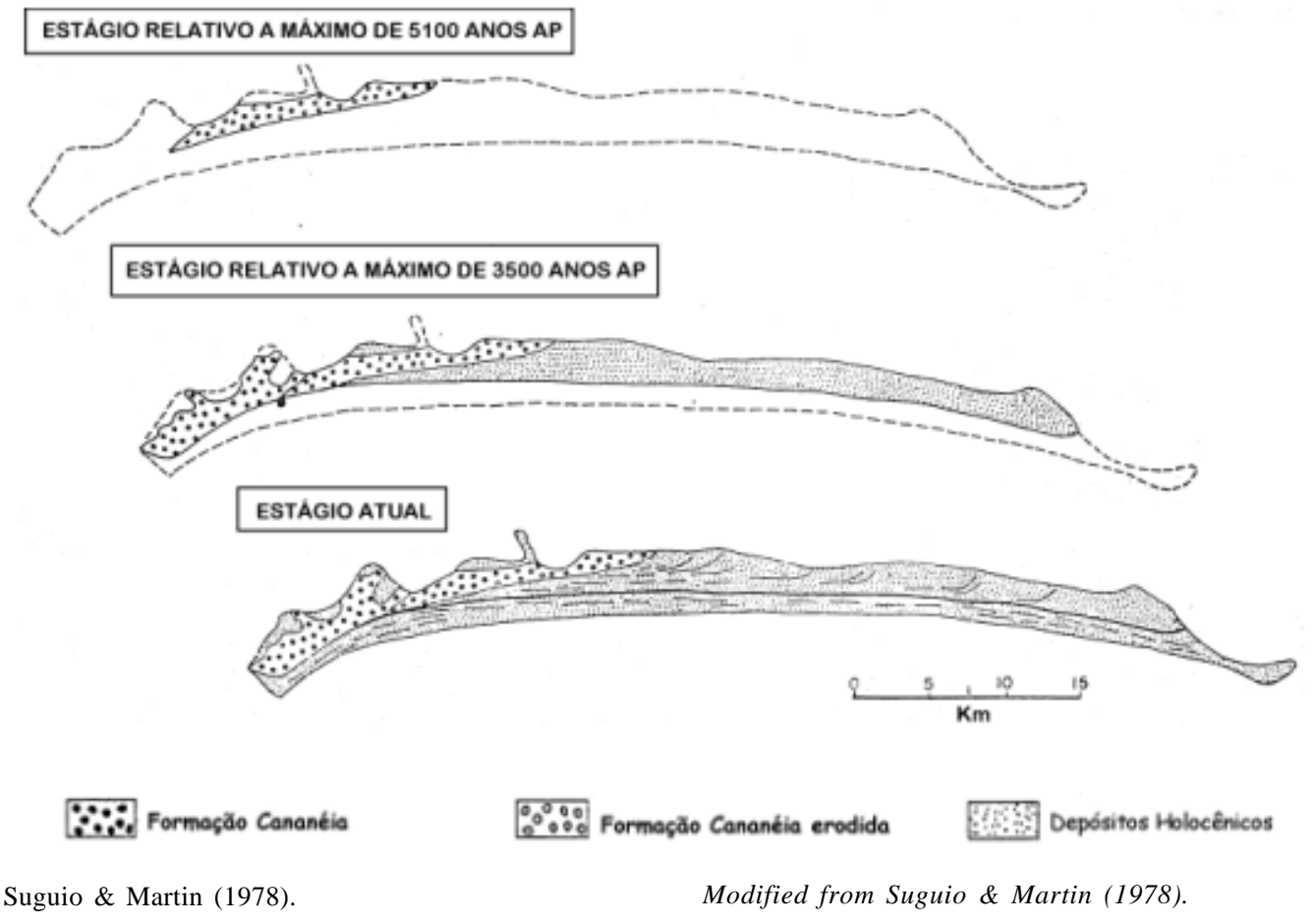

Figura 2: Evolução da Ilha Comprida durante o Holoceno.

Figure 2: Holocene evolution model of Ilha Comprida.

continente, bem como os cordões truncados e recurvados representativos das diversas etapas evolutivas da desembocadura do sistema interno em deslocamento para nordeste da Ilha.

Há aproximadamente 3.800 anos, a tendência geral de emersão da área sofreu uma interrupção quando então o nível do mar atingiu seu valor mínimo no período, voltando posteriormente, acerca de 3.500 anos, a atingir um outro máximo transgressivo. Após este pequeno máximo transgressivo, quando pode ter ocorrido a erosão dos cordões holocênicos internos, fenômeno este responsável pela formação da zona de baixa entre as duas gerações de cordões arenosos regressivos, a Ilha Comprida passou a apresentar um processo de alargamento pela adição de cordões paralelos a atual linha de costa. São desta fase os extensos e contínuos alinhamentos de cordões litorâneos da face externa da Ilha Comprida.

\section{O MÉTODO GPR}

O GPR é um método geofísico que utiliza ondas eletromagnéticas de altas frequiências $(10-1000 \mathrm{MHz})$ para a investigação detalhada de porções rasas do subsolo (até 50 metros de profundidade em condições favoráveis).
O método baseia-se na emissão de um pulso de energia eletromagnética para o subsolo através de uma antena transmissora, Tx. O sinal emitido sofre reflexões, refrações e difrações em descontinuidades presentes no meio de propagação e é então, captado ao retornar à superfície, por uma antena receptora, Rx (Fig. 3).

A propagação de uma onda eletromagnética de alta freqüência no terreno pode ser descrita pela sua velocidade e a atenuação sofrida durante o percurso. Em condições de baixa perda, a velocidade (v) está relacionada com a parte real da constante dielétrica (K) do meio de propagação, sendo dada pela seguinte expressão:

$$
\mathrm{v}=\frac{\mathrm{c}}{\sqrt{\mathrm{K}}}
$$

onde $c$ é velocidade da onda eletromagnética no espaço livre (Davis \& Annan, 1989).

As reflexões da energia eletromagnética ocorrem nas interfaces entre materiais ou camadas que apresentem distintos valores de velocidade. A amplitude do sinal refletido será mais intensa quanto maior for o contraste existente entre as velocidades (ou constantes dielétricas) dos correspondentes 


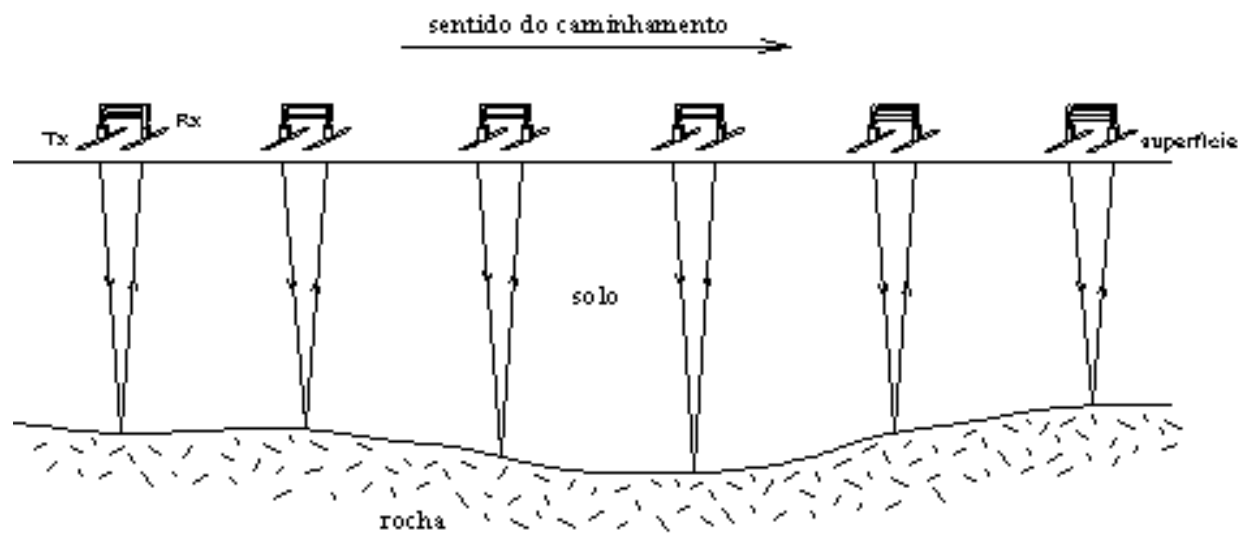

(Modificado de Davis \& Annan, 1989).

Figura 3 : Diagrama ilustrando a aquisição de dados GPR.
(Modified from Davis \& Annan, 1989).

Figure 3 : Diagram of GPR data acquisition system.

\begin{tabular}{||l|l|l|l|l||}
\hline MATERLAL & $\mathrm{K}$ & $\mathrm{V}(\mathbf{m} / \mathbf{n s})$ & $\mathbf{\sigma}(\mathbf{m S} / \mathbf{m})$ & $\mathbf{\alpha}(\mathbf{d B} / \mathbf{m})$ \\
\hline Ar & 1 & 0,3 & 0 & 0 \\
\hline Agua doce & 80 & 0,033 & 0,5 & 0,1 \\
\hline Agua do mar & 80 & 0,01 & 30000 & 1000 \\
\hline Areia seca & $3-5$ & 0,15 & 0,01 & 0,01 \\
\hline Areia saturada & $20-30$ & 0,06 & $0,1-1$ & $0,03-0,3$ \\
\hline
\end{tabular}

Tabela 1 - Valores característicos de K, v, $\sigma$ e $\alpha$ observados em diversos tipos de materiais para $100 \mathrm{MHz}$ (modificada de Davis \& Annan, 1989).

Table 1 - Common values for K, v, $\sigma$ and $\alpha$ observed in some materials for $100 \mathrm{MHz}$ antenna (modified from Davis \& Annan, 1989).

meios. A atenuação da onda está relacionada à condutividade elétrica dos materiais.

A Tab. 1 exibe alguns valores típicos da constante dielétrica $(\mathrm{K})$, velocidade $(\mathrm{v})$, condutividade $(\sigma) \mathrm{e}$ atenuação $(\alpha)$ para diversos tipos de materiais.

O GPR apresenta um ótimo desempenho em ambientes eletricamente resistivos (areias secas, por exemplo). Entretanto, uma boa qualidade do sinal pode também ser obtida em areias saturadas (Jol \& Smith, 1991; Neves et al., 1995). Em terrenos eletricamente condutivos (argilas em geral, água salgada, etc.) ocorre uma baixa penetração da onda eletromagnética em função da forte atenuação sofrida.

O sistema GPR pode operar com diversas freqüências, cada qual correspondendo a uma antena. A escolha da antena a ser utilizada depende do objetivo do levantamento (dimensões e profundidade do alvo) assim como das condições geológicas locais. Sinais de alta frequiência produzem alta resolução com pouca penetração, ocorrendo o inverso para sinais de baixa frequiência.

A aquisição dos dados é realizada efetuando-se medidas ao longo de um perfil mantendo constante o espaçamento entre as antenas (aquisição com afastamento comum). A rapidez com que o levantamento pode ser conduzido permite a obtenção de excelente cobertura espacial, produzindo imagens de alta resolução praticamente contínuas em subsuperfície. O tempo das reflexões representado em uma seção pode ser convertido em profundidade se conhecida a velocidade da onda eletromagnética no meio de propagação o que pode pode ser determinado no local através de ensaios específicos.

\section{DETERMINAÇÃO DA VELOCIDADE}

Para determinação da velocidade de propagação in situ, são utilizados os ensaios denominados Ponto Médio Comum (CMP) ou Reflexão e Refração de Grande Ângulo (WARR) que, através do progressivo aumento do espaçamento antena transmissora/ receptora possibilitam a obtenção do valor da velocidade característica do meio de propagação.

A Fig. 4 ilustra os eventos relacionados à reflexão e refração em um ensaio WARR, e o correspondente gráfico tempo vs. distância (domocrônica).

A hipérbole de reflexão observada no gráfico permite a determinação da velocidade através da correção do sobretempo normal (nmo) ou do método $\mathrm{t}^{2}-\mathrm{x}^{2}$ (Telford et al., 1990). Conhecendo-se o valor 


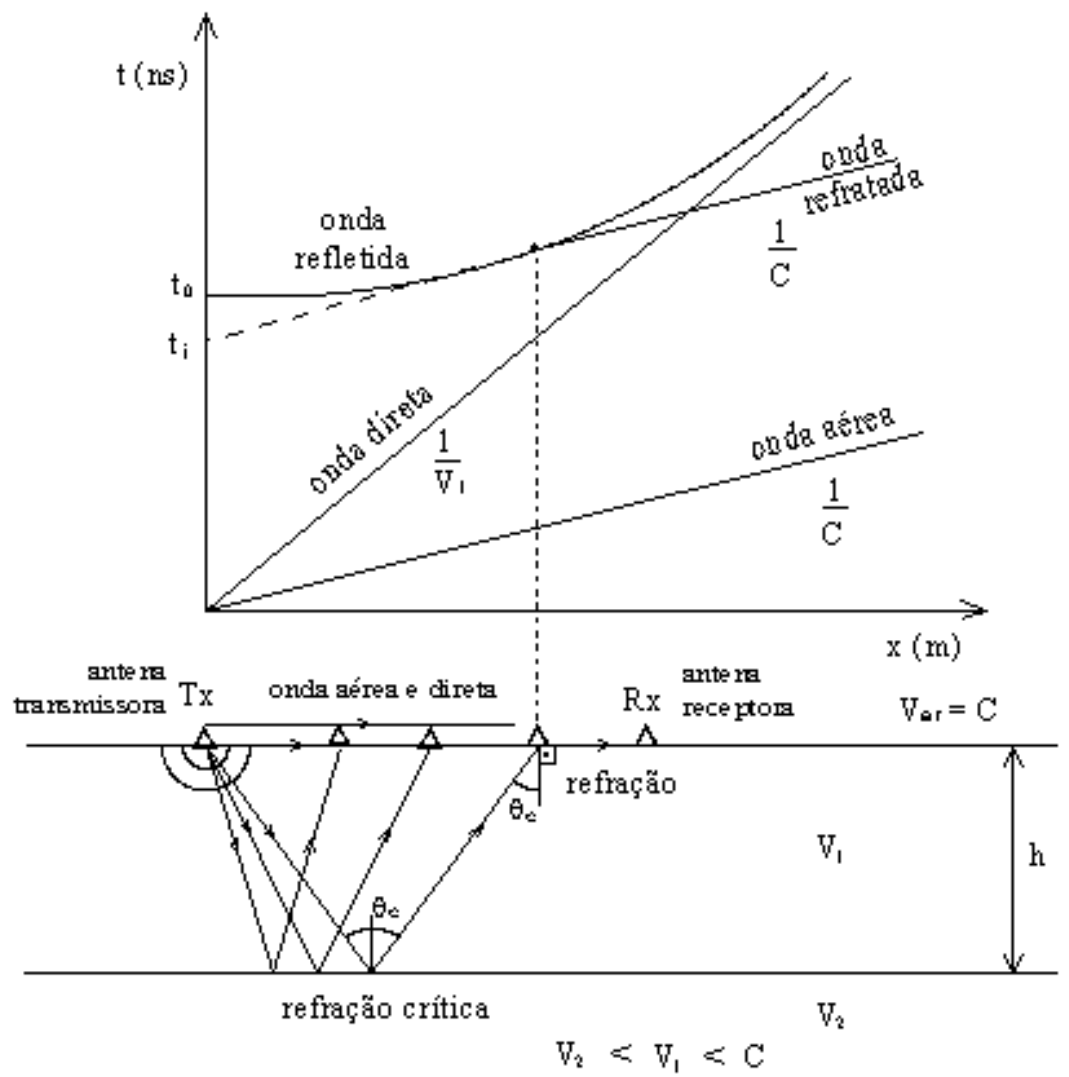

Figura 4- Diagrama esquemático com eventos observáveis num ensaio WARR.

Figure 4-Schematic view of WARR events.

da velocidade por estes métodos, a profundidade do refletor $(\mathrm{h})$ pode ser calculada através de:

$$
\mathrm{h}=\frac{\mathrm{v}_{1} \cdot \mathrm{t}_{0}}{2},
$$

onde $\mathrm{v}_{1}$ é a velocidades da onda na primeira camada e $t_{0}$ é o "tempo zero", que equivale ao tempo da reflexão em uma situação de afastamento nulo entre as antenas.

A identificação das ondas refratadas na interface solo/ar pode também auxiliar no cálculo da profundidade utilizando-se as seguintes expressões:

$$
\begin{gathered}
\theta_{\mathrm{c}}=\operatorname{sen}^{-1}\left(\frac{\mathrm{v}_{1}}{\mathrm{v}_{\mathrm{ar}}}\right)=\operatorname{sen}^{-1}\left(\frac{\mathrm{v}_{1}}{\mathrm{c}}\right), \\
\mathrm{h}=\frac{\mathrm{v}_{1} \cdot \mathrm{t}_{\mathrm{i}}}{2 \cdot \cos \theta_{\mathrm{c}}}=\frac{\mathrm{t}_{\mathrm{i}} \cdot \mathrm{v}_{1} \cdot \mathrm{v}_{\mathrm{ar}}}{2 \cdot \sqrt{\mathrm{v}_{\mathrm{ar}}^{2}-\mathrm{v}_{1}^{2}}}=\frac{\mathrm{t}_{\mathrm{i}} \cdot \mathrm{v}_{1} \cdot \mathrm{c}}{2 \cdot \sqrt{\mathrm{c}^{2}-\mathrm{v}_{1}^{2}}},
\end{gathered}
$$

onde $\mathrm{v}_{\mathrm{ar}}=\mathrm{c}$ é a velocidade da onda no espaço livre (ar), $t_{i}$ é o tempo de intercepção e $\theta_{c}$ é o ângulo da refração crítica.

O conhecimento da velocidade da onda permite a conversão tempo-profundidade e a aplicação da técnica de migração, na qual a velocidade constitui o principal parâmetro de entrada. A migração dos dados tem por objetivo o deslocamento espacial, posicionando os refletores em suas corretas posições em subsuperfície, uma vez que a seção de afastamento comum apresenta uma imagem distorcida. Pestana \& Botelho (1997) apresentam um exemplo onde foi aplicado a migração de dados GPR com correção topográfica simultânea em ambiente geológico similar ao que foi desenvolvido neste trabalho.

\section{EQUIPAMENTO E AQUISIÇÃO DOS DADOS}

O equipamento empregado no levantamento de campo foi o modelo RAMAC/GPR (MALÄ GeoScience), utilizando-se as antenas de 50 e 100 MHz.

Na aquisição com afastamento comum utilizando a antena de $100 \mathrm{MHz}$, os dados foram coletados de maneira contínua, com espaçamento entre pontos de medida igual a 1 metro, 32 leituras em cada ponto. As antenas foram montadas sobre um dispositivo rebocado por um veículo, permitindo uma aquisição 
extremamente rápida, onde os dados puderam ser registrados automaticamente (Fig. 5). Foram adquiridos 1732 traços, com 512 amostras por traço que, na freqüência de amostragem igual a $1006 \mathrm{MHz}$, corresponde a uma janela de 509 ns. Segundo Davis \& Annan (1989) a utilização de antenas com freqüência central de aproximadamente $100 \mathrm{MHz}$ são aquelas que fornecem o melhor compromisso entre penetração, resolução e portatibilidade nos mais diversos ambientes geológicos.

O levantamento com a antena de $50 \mathrm{MHz}$ foi conduzido de maneira não contínua, com espaçamento entre pontos de leitura igual a 1 metro, com 64 medidas em cada ponto e antenas separadas por uma distância de 2 metros (Fig. 6). Foram adquiridos 1287 traços, com 512 amostras por traço que, na freqüência de amostragem igual a $500 \mathrm{MHz}$, corresponde a uma janela de $1024 \mathrm{~ns}$.

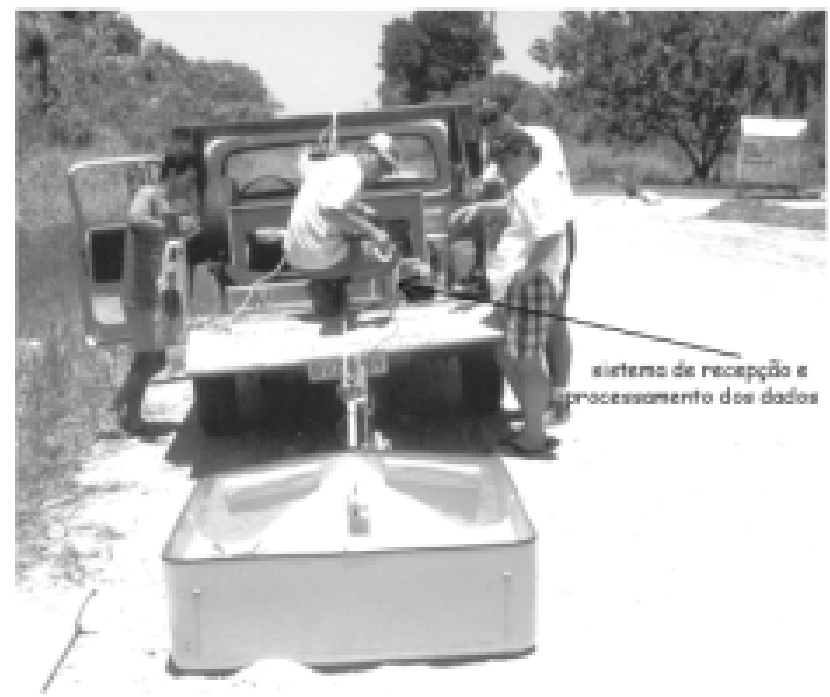

Figura 5 - Operação de campo para aquisição de dados com a antena de $100 \mathrm{MHz}$.

Figure 5 - Field works for data acquisition with $100 \mathrm{MHz}$ antenna.

O ensaio WARR foi realizado com a antena de $50 \mathrm{MHz}$, de modo a garantir uma boa penetração do sinal. O ponto escolhido para a realização deste ensaio foi aproximadamente a posição $600 \mathrm{~m}$ no perfil. $\mathrm{O}$ espaçamento entre medidas foi de $0,2 \mathrm{~m}$ com 128 leituras em cada ponto.

\section{PROCESSAMENTO DOS DADOS}

No processamento dos dados foram utilizados os programas computacionais RADPRO (v.2.25, MALÄ GeoScience, Suécia) e GRADIX (v.1.11,

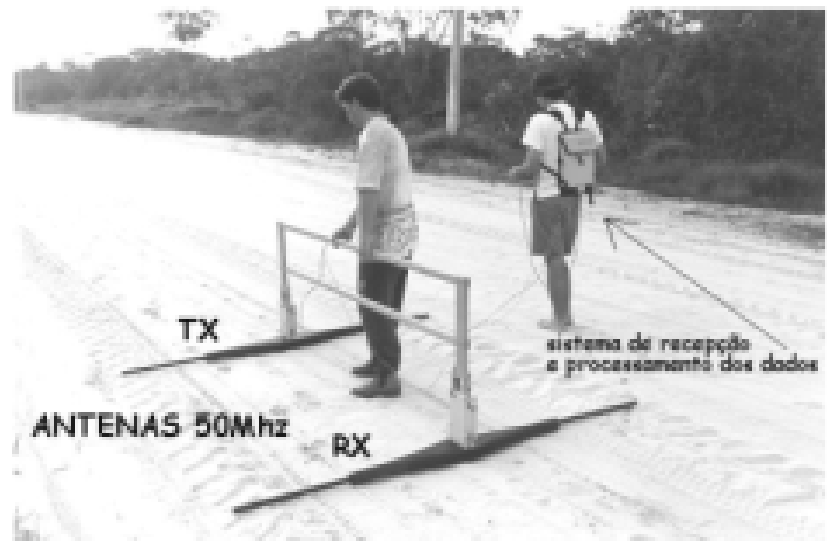

Figura 6 - Operação de campo para aquisição de dados com a antena de $50 \mathrm{MHz}$.

Figure 6-Field works for data acquisition with $50 \mathrm{MHz}$ antenna.

Interpex Limited, USA) que são aplicativos dedicados ao tratamento de dados GPR.

As seções obtidas em campo foram processadas adotando-se os seguintes procedimentos: correção do tempo-zero e remoção da componente DC, aplicação de ganho (AGC), filtragem temporal (passabanda trapezoidal), conversão tempo-profundidade e migração.

A seção WARR obtida é apresentada na Fig. 7. Pode ser observado que a hipérbole assinalada em $\mathrm{t}_{\mathrm{o}}=260 \mathrm{~ns}$ (indicada por uma seta na figura) está relacionada ao forte refletor plano com considerável extensão lateral e bem nítido nas seções de afastamento constante (entre 500 e 700 metros, no tempo de aproximadamente $260 \mathrm{~ns}$, Figs. 8 e 9 ) adquiridas com as antenas de 50 e $100 \mathrm{MHz}$.

A correção do sobretempo normal e o método $\mathrm{t}^{2}-\mathrm{x}^{2}$, fornecem um valor de velocidade igual a 0,07 $\mathrm{m} / \mathrm{ns}$ para o pacote situado acima do refletor que, de acordo com a eq.(2), encontra-se a aproximadamente a 9 m de profundidade:

$$
\mathrm{h}=\frac{\mathrm{v}_{1} \cdot \mathrm{t}_{0}}{2}=\frac{0,07 \cdot 260}{2}=9,1 \mathrm{~m}
$$

Observa-se de fato que a hipérbole tende assintoticamente à reta relacionada à onda direta, que por sua vez também apresenta velocidade aparente igual a $0,07 \mathrm{~m} / \mathrm{ns}$, representando as porções superficiais do subsolo.

A hipérbole observada no tempo $t_{0}=520 \mathrm{~ns}$ equivale a uma múltipla da reflexão primária, de acordo com os argumentos apresentados por Gandolfo (1999): 1) O tempo zero da suposta múltipla é exatamente o dobro do correspondente à reflexão 


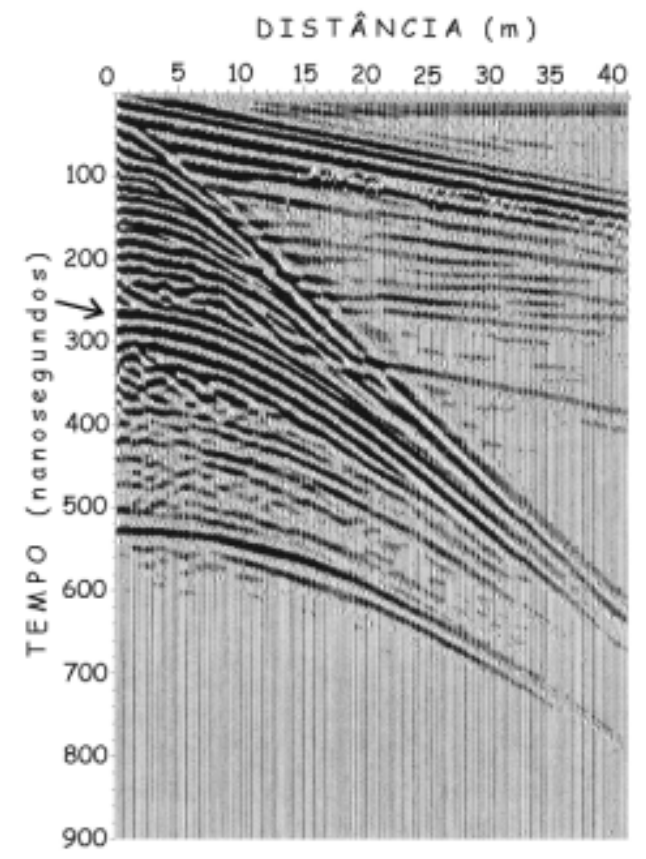

Figura 7- Registro WARR obtido na área.

Figure 7-WARR profile obtained in the studied area. primária $\left(t_{\mathrm{o}}=260 \mathrm{~ns}\right)$. 2) A velocidade que corrige $\mathrm{o}$ sobretempo normal da primeira hipérbole, também corrige o da segunda. 3) Ocorre uma inversão de polaridade entre os eventos mencionados.

A condição favorável do ambiente geológico possibilitou a identificação da onda refratada na interface solo/ar. Este evento pode fornecer informações úteis e complementares para a investigação da subsuperfície (Bohidar \& Hermance, 2002).

No gráfico tempo vs. distância, este evento com velocidade aparente igual a $0,3 \mathrm{~m} / \mathrm{ns}$ exibe paralelismo à reta relacionada com a onda direta que caminha pelo ar. Da seção WARR ainda é possível obter o tempo de intercepção, t (igual a $250 \mathrm{~ns}$ ), e determinar o ângulo crítico da refração (Eq.3):

$$
\theta_{c}=\operatorname{sen}^{-1}\left(\frac{v_{1}}{v_{a r}}\right)=\operatorname{sen}^{-1}\left(\frac{0,07}{0,3}\right)=13,5^{\circ},
$$

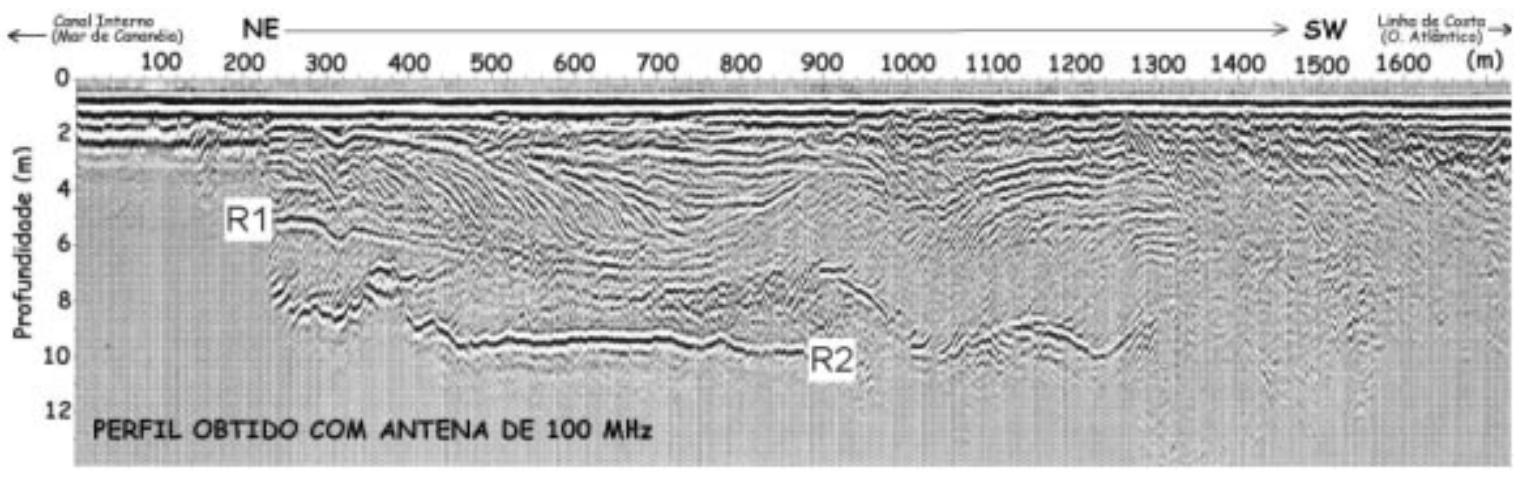

Figura 8: Perfil GPR obtido com a antena de $100 \mathrm{MHz}$

Figure 8: GPR profile from 100MHz antenna.

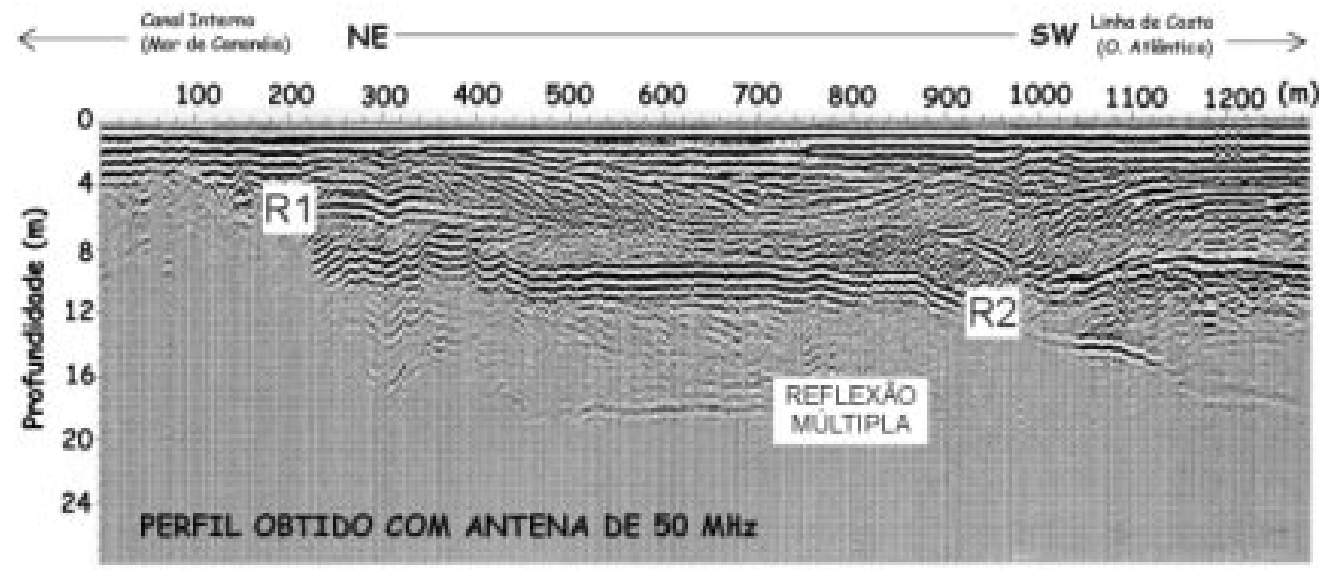

Figura 9: Perfil GPR obtido com a antena de $50 \mathrm{MHz}$

Figure 9: GPR profile from 50MHz antenna. 
e ainda a profundidade do refletor através da Eq.(4)

$$
h=\frac{v_{1} \cdot t_{i}}{2 \cdot \cos \theta_{c}}=\frac{t_{i} \cdot v_{1} \cdot v_{a r}}{2 \cdot \sqrt{v_{a r}^{2}-v_{1}^{2}}}=9,0 \mathrm{~m}
$$

Estes valores estão em plena concordância com os determinados anteriormente através da correção do sobretempo normal e do método $\mathrm{t}^{2}-\mathrm{x}^{2}$, mostrando grande coerência entre os resultados obtidos.

Utilizando o valor da velocidade determinado para o meio, foi possível uma confiável conversão tempo-profundidade e a execução da migração dos dados.

As Figs. 8 e 9 apresentam as seções finais correspondentes, respectivamente, aos perfis levantados com a antena de 100 e $50 \mathrm{MHz}$, onde o refletor plano encontra-se, de fato, posicionado a aproximadamente 9 metros de profundidade.

\section{ANÁLISE E INTERPRETAÇÃO DOS DADOS}

A análise dos registros de GPR obtidos na região sul da Ilha Comprida permitiu identificar a existência de duas seqüências distintas, uma composta por refletores formando estruturas indicativas de um processo de progradação, em sentido SW, sobreposta a outra de características reflectivas semelhantes, se distinguindo apenas pela ausência de padrões estruturais de geometria identificável. Destaca-se ainda um forte refletor que ocorre na base deste conjunto. Para uma análise interpretativa dos registros neste trabalho, o limite entre estas duas sequiências fica definido como R1 e o refletor da base dos registros como R2. Estas feições estão indicadas nas Figs. 8 e 9.

Com base no modelo evolutivo da Ilha Comprida, proposto por Suguio \& Martin (1978) e através da análise de velocidade de propagação das ondas eletromagnéticas, os registros indicam a presença de um pacote extremamente homogêneo entre a superfície e 9,1 metros de profundidade, neste caso constituído por sedimentos arenosos, possivelmente saturados, pois a velocidade determinada $(0,070 \mathrm{~m} /$ ns) é muito baixa para representar areias secas (Tab. 1). Resultado similar pode ser verificado em ambiente arenoso e com nível d'água raso (Daly et al., 2002).

Dessa forma, o refletor situado a 9,1 metros de profundidade (Fig. 8) não corresponderia ao nível d'água já que, informações levantadas na região indicam que os poços rasos têm o nível do lençol freático bastante raso (entre 1,0 m e 1,5 m) e oscilante em função das marés, que têm variações médias em torno de 1,5 metro na área.

Este refletor corresponderia ao topo de uma camada que apresenta propriedades eletromagnéticas distintas em relação às seqüências superiores. Abaixo desta interface observa-se a ausência de sinal (sombra) no registro, indicando que toda a energia incidente ou foi refletida neste contato ou atenuada/ absorvida de forma mais intensa sob este refletor. Este limite marcante, definido nos registros como R2, pode ser representativo de condições ambientais bastante distintas, dentro dos processos sedimentares que regeram a formação da Ilha Comprida, associadas às variações relativas do nível médio do mar durante o Quaternário.

Numa correlação estratigráfica entre o radargrama e o modelo evolutivo citado, a partir do refletor de base (R2), pode-se considerar que ele representaria uma superfície de erosão da seqüência de areias e argilas transgressivas pleistocênicas do evento de elevação do nível médio do mar de 120.000 anos A.P., que atingiu cotas de aproximadamente 8 metros e sobre a qual foram formados cordões arenosos na fase regressiva, conforme descritos em Suguio \& Martin (1978).

Parte desses cordões foram erodidos e retrabalhados no evento transgressivo holocênico, sendo que, a presença dos cordões arenosos retrogradantes identificados nas porções superiores do registro estaria relacionada ao evento holocênico regressivo subseqüiente (5.100 A.P.), responsável pelo processo de alargamento da Ilha Comprida conforme estabelecido por Suguio \& Martin (op cit.). O contato entre estas duas seqüências corresponderia nos registros do radar ao refletor $\mathrm{R} 1$, que separa essas duas unidades de características distintas.

Dentro do contexto das características geológicas da região da Ilha Comprida, o refletor R2 poderia representar, não apenas o contato erosivo entre as seqüências sedimentares do Pleistoceno e as formadas durante o evento Holocênico mas também o registro de camadas argilosas ricas em matéria orgânica, depositadas nos vales formados entre dois cordões arenosos adjacentes. 


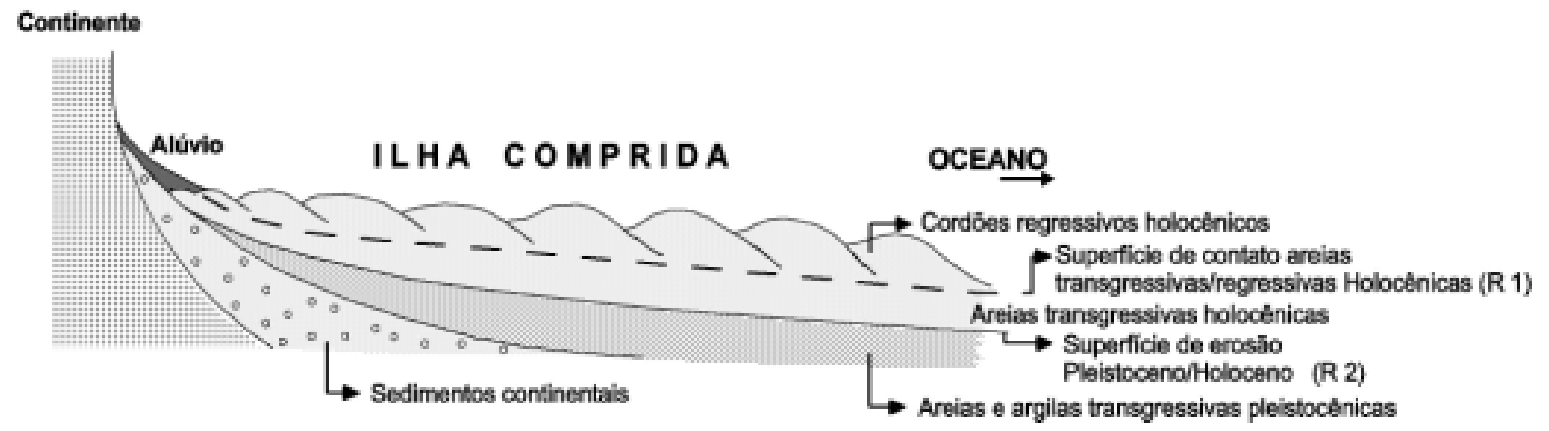

Figura 10: Estágio 2 do modelo evolutivo esquemático para a Ilha Comprida proposto por Suguio \& Martin (1978).

Figure 10: Ilha Comprida recent evolution geologic model - Phase 2 from Suguio \& Martin (1978).

Existe ainda a possibilidade deste refletor corresponder a uma superfície endurecida por um processo pedogenético, superficial, em que os sedimentos arenosos são compactados e cimentados pela percolação de ácidos húmicos, originando uma rocha endurecida e muito pouco permeável, localmente denominada de Piçarra presente nos sedimentos holocênicos.

Muito embora não existam dados de testemunhos de sondagem que permitam uma correlação direta entre o refletor identificado com as hipóteses sugeridas, a existência na área de superfícies de contato erosivo das seqüências holocênicas/ pleistocênicas (Suguio \& Tessler, 1987), bem como da ocorrência de Piçarras na área, dão suporte às idéias apresentadas.

Analisando a representação esquemática do modelo evolutivo proposto por Suguio \& Martin (1978) apresentada na Fig. 10, e integrando-a ao registro interpretado da Fig 11, pode-se situá-los estratigraficamente, sendo que eles compreenderiam as sequiências denominadas "areias transgressivas holocênicas" e "cordões regressivos holocênicos". Os refletores R2 e R1 corresponderiam ao contato entre as areias e argilas transgressivas pleistocênicas e as areias transgressivas holocênicas e, a superfície de erosão entre as areias transgressivas holocênicas e os cordões regressivos holocênicos, respectivamente.

\section{CONCLUSÕES}

A evolução da planície costeira do sistema Cananéia Iguape é resultado da alternância de seqüências transgressivas e regressivas durante o Quaternário e suas características são resultados da interação dos parâmetros geológicos-geomorfológicos e dos processos dinâmicos que agem continuamente durante esses grandes eventos (Suguio \& Martin, 1978).

A identificação dos registros sedimentares associados a esses processos, através de método indireto, especificamente o GPR confirmou, através

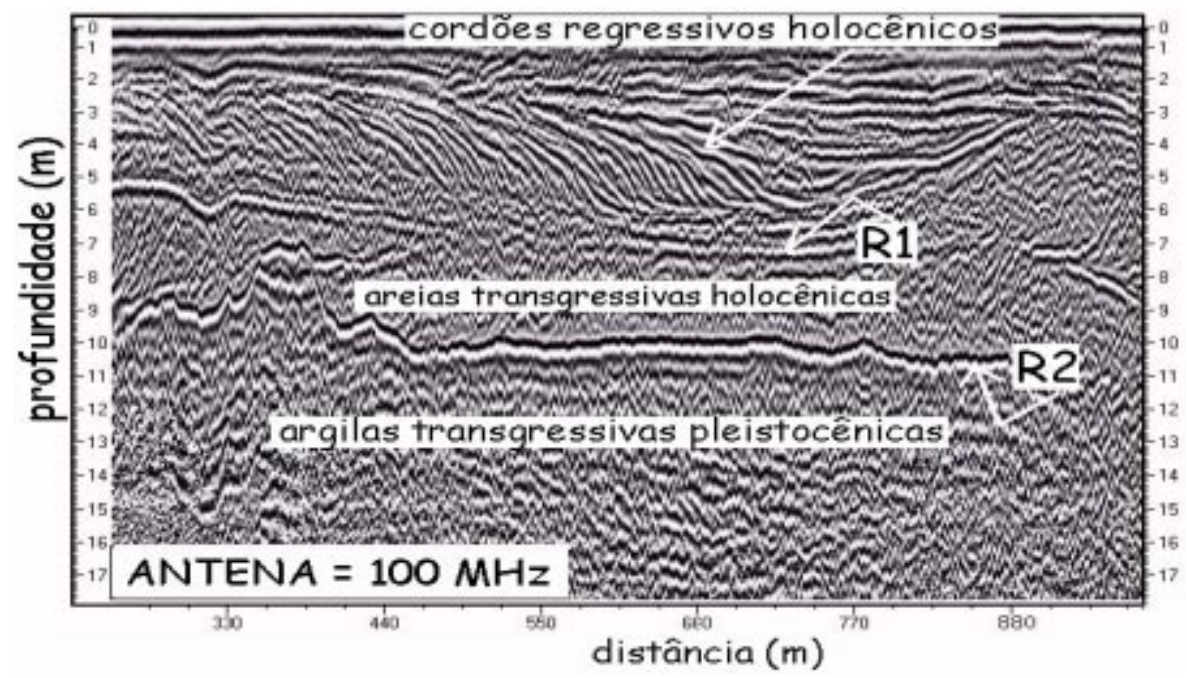

Figura 11 - Registro interpretado de acordo com o modelo da Fig.10 Figure 11 - Interpreted GPR section according to model of Fig.10. 
dos ensaios realizados na Ilha Comprida, a enorme potencialidade deste tipo de abordagem, no estudo detalhado da estratigrafia rasa em áreas costeiras continentais. Conforme pode ser observado nas seções obtidas deste levantamento, detalhes de dimensões decimétricos-centimétricos, podem ser analisados. Dessa forma, foi possível estabelecer uma correlação estratigráfica entre os registros do GPR e as informações de campo citadas em literatura, caracterizando as estruturas internas das sequiências sedimentares descritas por Suguio \& Martin, (1978).

Os limites destas sequiências, da base para o topo, corresponderiam aos refletores $\mathrm{R} 2 \mathrm{e} \mathrm{R} 1$, sendo que o primeiro corresponderia a uma superfície de erosão dos depósitos pleistocênicos, compostos por areias transgressivas do evento de elevação do nível do mar de 120.000 anos A.P.. O refletor 1 estaria associado ao contato entre a sequiência transgressiva holocênica, que erodiu e retrabalhou os sedimentos pleistocênicos, com as areias do evento regressivo subseqüente, de 5100 anos A.P., onde se pode observar, através do radargrama, estruturas retrogradantes que confirmam a relação entre o evento e o alargamento da Ilha.

A possibilidade de observação da correlação entre os refletores e pacotes sedimentares obtidos nos registros do GPR com as seqüências sedimentares descritas em literatura e qualidade dos resultados obtidos, enfatizam a viabilidade da aplicação deste tipo de método investigativo. Pode-se considerar sua aplicabilidade não apenas neste tipo de estudo, mas também em estudos aplicados a obras civis, gerenciamento ambiental e demais atividades onde seja necessário o levantamento de informações imediatas sobre o substrato raso em função de este método ter grande flexibilidade operacional, sendo possível cobrir extensas áreas, com grande detalhe e num curto período de tempo, quando comparado a qualquer outro método geofísico de investigação.

\section{REFERÊNCIAS}

Barcelos, J. H., 1975. Sedimentação e subambientes deposicionais da Ilha Comprida, São Paulo. Dissertação de Mestrado, IG/USP: 155pp.

Beres Jr. M. \& Haeni, F. P., 1991. Application of ground - penetrating - radar methods in hydrogeologic studies. Ground Water. 29(3): 375386.

Bohidar, R. N. \& Hermance, J. F., 2002. The GPR refraction method. Geophysics. 67(5): 14741485.

Daly, J., McGeary S. \& Krantz, D. E., 2002. Ground Penetrating Radar Investigation of a Late Holocene Spit Complex: Cape Henlopen, Delaware. Journal of Coastal Research. 18(2): 274-286.

Davis, J. L. \& Annan, A. P., 1989. Groundpenetrating radar for high-resolution mapping of soil and rock stratigraphy. Geophysical Prospecting. 37(5): 531-551.

Fisher, E., McMechan, G. A. \& Annan, A. P., 1992. Acquisition and processing of wideaperture ground-penetrating radar data. Geophysics. 57(3): 495-504.

Gandolfo, O. C. B., 1999. Aplicação da sísmica de reflexão de alta resolução e do radar de penetração no solo (GPR): um estudo comparativo. Dissertação de Mestrado, IGCE/ UNESP:140pp.

Jol H. M. \& Smith D. G., 1991. Ground penetrating radar of northern lacustrine deltas. Canadian Journal of Earth Sciences. 28: 1939-1947.

Jol H. M., Smith D. G. \& Meyers R. A., 1996. Digital Ground Penetrating Radar (GPR): A New Geophysical Tool for Coastal Barrier Research (Examples from the Atlantic, Gulf and Pacific Coasts, U.S.A). Journal of Coastal Research. 12(4): 960-968.

Neves, F. A., Roulston, M. S. \& Miller, J. A., 1995. Source signature deconvolution of ground penetrating radar data. Revista Brasileira de Geofísica. 13(2): 143-153.

Pestana, R. C. \& Botelho M. A. B., 1997. Migração de dados de radar (GPR) com correção topográfica simultânea. Revista Brasileira de Geofísica. 15(1): 3-10.

Suguio, K. \& Martin, L., 1978. Formações quaternárias marinhas do litoral paulista e sul fluminense. In: International Symposium on Coastal Evolution in the Quaternary. São Paulo 1978. Special Publication $n^{\circ} 1$. The Brazilian National Working Group for the IGCP Project 61, IG/USP, SBG: 55pp. 
Suguio, K. \& Tessler, M. G., 1987. Characteristics of a Pleistocene nearshore deposits: An example from southern São Paulo State coastal plain. In Quaternary of South America And Antartica Peninsula (Jorge Rabassa, ed.). A. A. Balkema/ Rotterdan/Brookfield. 5: 257-267.
Telford, W. M., Geldart, L. P. \& Sherif, R. E., 1990. Applied Geophysics. 2ed. Cambridge: Cambridge University Press, 770pp.

Van Overmeeren, R. A., 1997. Radar facies of unconsolidated sediments in The Netherlands: A radar stratigraphy interpretation method for hydrogeology. Journal of Applied Geophysics. 40: $1-18$.

\section{NOTES ABOUT THE AUTHORS}

\section{Otávio Coaracy Brasil Gandolfo}

Físico pela PUC-SP. Geofísico pelo Instituto Astronômico e Geofísico da USP. Mestre em Geociências e Meio Ambiente pelo IGCE-UNESP. Doutorando no Instituto de Geociências da USP. Atua na área de geofísica aplicada.

\section{Luiz Antonio Pereira de Souza}

Geólogo do Laboratório de Geofísica Aplicada do Instituto de Pesquisas Tecnológicas do Estado de São Paulo - IPT, desde 1981. Doutorando em oceanografia geológica no Programa de Pós Graduação do Instituto Oceanográfico da USP. Atua na área de geofísica aplicada à investigação de áreas submersas.

\begin{abstract}
Moysés Gonsalez Tessler
Professor Doutor do Departamento de Oceanografia Física do Instituto Oceanográfico da USP desde 1979. Desenvolveu Mestrado, Doutorado e Livre Docência na área da oceanografia geológica, mais diretamente em processos de dinâmica sedimentar de áreas costeiras e de plataforma continental.

Marcelo Rodrigues

Geólogo do Grupo de Oceanografia Geológica do Departamento de Oceanografia Física (DOF) do Instituto Oceanográfico da USP desde 1995. Mestre em Oceanografia Geológica. Atua na área de oceanografia geológica e geofísica marinha.
\end{abstract}

INSTITUTO DE PES QUISAS TECNOLÓGICAS DO ESTADO DE S ÃO PAULO LABORATÓRIO DE GEO FÍS ICA APLICADA DO IPT

The Applied Geophysics Laboratory (LGA) ope rates in the sub are as of enginee ring ge ology, hydrogeology, environment and mine ral prospects and for that makes use of the following geophysical me thods:

- Seismic methods: reflection, refraction, crosshole, continuous se ismic profiling and sonography;

- Electric and electromagne tic me thods:electro-resistivity, spontaneous potential, induced

polarization, electromagne tome try, tomography and GPR(Ground Pe ne trating Radar);

- Potential me thods:gravime try and magne tometry

\section{MONTE CARLOSIMULATIONOFNUCLEARLOGGINGDETECTIONSYSTEMS NOTE ABOUT THE AUTHOR}

\section{Jadir C. da Silva}

Bsc (Civil and Transport Engineer) at the Escola de Engenharia Kennedy, Belo Horizonte, MG, 1985.

Msc (Nuclear Science and Thecnology) at the Universidade Federal de Minas Gerais, Belo Horizonte, MG, 1988.
$\mathrm{PhD}$. (Geophysics) at the Universidade Federal do Pará, Belém, PA, 1993.

Research Activities: Professor of Geophysics at the Universidade Federal do Rio de Janeiro, since 1998. Areas of interest: Well-logging and Applied Geophysics modeling. 